\section{Prevalência de transtornos mentais comuns e contexto social: análise multinível do São Paulo Ageing \& Health Study (SPAH)}

\author{
Prevalence of common mental disorders and \\ the relationship to the social context: multilevel \\ analysis of the São Paulo Ageing \& Health Study \\ (SPAH)
}

\section{Prevalencia de trastornos mentales comunes y contexto social: análisis multinivel del São Paulo Ageing \& Health Study (SPAH)}

\begin{abstract}
Social context can play a important role in the etiology and prevalence of mental disorders. The aim of the present study was to investigate risk factors for common mental disorders (CMD), considering different contextual levels: individual, household, and census tract. The study used a population-based sample of 2,366 respondents from the São Paulo Ageing \& Health Study. Presence of CMD was identified by the SRQ-20. Sex, age, education, and occupation were individual characteristics associated with prevalence of CMD. Multilevel logistic regression models showed that part of the variance in prevalence of CMD was associated with the household level, showing associations between crowding, family income, and CMD, even after controlling for individual characteristics. These results suggest that characteristics of the environment where people live can influence their mental health status.
\end{abstract}

Mental Disorders; Mental Health; Multilevel Analysis
Letícia Maria Silva Coutinho 1 Alícia Matijasevich 1 Márcia Scazufca 1 Paulo Rossi Menezes 1

\section{Resumo}

O contexto social pode ter papel importante na etiologia dos transtornos mentais e na sua prevalência. O objetivo do presente estudo foi investigar fatores de risco que contribuem para a prevalência de transtornos mentais comuns (TMC), considerando distintos níveis contextuais: indivíduo, domicílio e setor censitário. Para isso, utilizou-se uma amostra de base populacional de 2.366 indivíduos participantes do São Paulo Ageing \& Health Study. A presença de TMC foi identificada pelo instrumento SRQ-20. Sexo, idade, escolaridade e ocupação foram características individuais associadas à prevalência de TMC. Modelos de regressão logística multinível mostraram que parte da variância na prevalência de TMC foi associada ao nível do domicílio, com associações entre aglomeração, renda familiar e prevalência de TMC, mesmo após controle para características individuais. Esses resultados sugerem que características do ambiente onde as pessoas vivem contribuem para sua saúde mental.

Transtornos Mentais; Saúde Mental; Análise Multinível 


\section{Introdução}

Problemas de saúde mental são responsáveis por uma morbidade significativa em todo o mundo 1, atingindo, aproximadamente, 700 milhões de pessoas e representando um terço do total de casos de doenças não transmissíveis, segundo a Organização Mundial da Saúde (OMS). Cerca de $90 \%$ das pessoas com problemas de saúde mental apresentam manifestações de depressão e ansiedade, incluindo sintomas como insônia, fadiga, irritabilidade, dificuldade de memória e concentração e queixas somáticas ${ }^{2}$. O termo "transtorno mental comum" (TMC) tem sido utilizado para designar situações de saúde em que o indivíduo apresenta sintomas de depressão e/ou ansiedade em intensidade suficiente para interferir em suas atividades diárias, sem que necessariamente sejam preenchidos os critérios formais para esses diagnósticos segundo as classificações atuais, como o Manual Diagnóstico e Estatístico de Transtornos Mentais, 4a edição (DSM-IV) e a 10a revisão da Classificação Estatística Internacional de Doenças e Problemas Relacionados à Saúde (CID-10) 3,4,5. Os TMC são mais prevalentes entre mulheres e pessoas com piores condições socioeconômicas, e a pobreza e o desemprego aumentam a duração de episódios de TMC 6 .

Estudos recentes têm sugerido que o contexto social, como, por exemplo, o local onde as pessoas residem, também, pode ter um papel na etiologia e no prognóstico dos TMC. Em estudo realizado no País de Gales, Skapinakis et al. 7 encontraram associação entre área de residência e escores de sofrimento mental. Utilizando uma análise multinível, os autores observaram que $1,47 \%$ da variância nos escores de sofrimento mental era explicada pela área de residência. Em estudo realizado em Santiago (Chile), Araya et al. 8 examinaram a associação entre aspectos da qualidade do ambiente, como residir em ruas asfaltadas e maior quantidade de áreas verdes, e a saúde mental dos moradores. Os resultados evidenciaram uma associação inversa entre qualidade do ambiente e presença de TMC. Em outra análise, Araya et al. ${ }^{9}$ investigaram a relação entre saúde mental e capital social. Após ajuste para variáveis individuais, dois componentes do capital social, confiança e coesão social mostraramse associados com escores de saúde mental. No entanto, esses fatores mostraram pouca variação no nível contextual, sugerindo uma maior influência das características individuais na saúde mental da população. No entanto, investigação conduzida no País de Gales por Thomas et al. 10 sugere que características do domicílio onde os indivíduos residem podem ter maior relevância na ocorrência de TMC do que características da área de residência. Weich et al. 11 encontraram resultados semelhantes em análise de uma amostra representativa do Reino Unido. Menos de $1 \%$ do total da variância de TMC foi explicada pelo nível dos setores censitários, 4,5\% foram explicados pelo nível dos domicílios, enquanto a maior parte da variância foi explicada pelo nível individual.

Até o presente, são escassas as evidências sobre a associação entre contexto social e prevalência de TMC em países de renda média e baixa. É possível que no Brasil, país com grandes centros urbanos e marcadas disparidades sociais, o contexto social possa ter um impacto ainda maior na prevalência de TMC que nos países ricos. A cidade de São Paulo, com 11,2 milhões de habitantes, onde grande parte da população vive nas periferias e em regiões centrais deterioradas, é um cenário interessante para esse tipo de investigação ${ }^{12}$. O presente trabalho tem como objetivo investigar a contribuição de fatores contextuais, representados por dois níveis distintos, setores censitários e domicílios, na prevalência de TMC.

\section{Método}

\section{Desenho do estudo e amostra}

A investigação epidemiológica São Paulo Ageing \& Health Study (SPAH) é um estudo de base populacional com delineamento de corte transversal em sua primeira fase, realizado para investigar a saúde de idosos com 65 anos ou mais que vivem em áreas de baixa renda da região oeste da cidade de São Paulo. Detalhes sobre a amostra do SPAH foram publicados previamente 13. De forma resumida, foram identificadas e convidadas a participar da primeira fase do SPAH todas as pessoas de 65 anos ou mais, residentes em 66 setores censitários da região oeste do Município de São Paulo. Das 2.226 pessoas potencialmente elegíveis, 2.072 consentiram em participar do estudo. Para cada idoso incluído no SPAH, foi também identificado e incluído um informante. Os informantes eram parentes ou amigos próximos aos idosos, com, no mínimo, 16 anos de idade, que, na maioria dos casos, residiam com os idosos participantes. Os idosos participantes do SPAH e seus informantes foram avaliados em relação à presença de sintomas de TMC. Para a presente análise, identificamos os domicílios com, pelo menos, duas pessoas (idosos e seus informantes) que possuíssem a medida de desfecho de interesse (TMC). Dessa maneira, a amostra da presente análise foi composta por 2.366 pessoas $(1.427$ idosos e 939 informantes) residentes em 1.148 
domicílios de 61 setores censitários. O maior número de idosos em relação ao número de informantes deve-se a situações em que dois ou mais idosos tinham um mesmo informante ou situações nas quais dois ou mais idosos residiam no mesmo domicílio e um era o informante do outro. As avaliações foram realizadas nos domicílios dos participantes.

\section{Medidas de desfecho e de exposição}

O desfecho foi definido como presença de TMC, identificada pelo instrumento SRQ-20 (Self Reporting Questionnaire), questionário composto por 20 itens, com respostas tipo "sim" ou "não" 14. O escore total do SRQ varia de 0 a 20 , com escores mais elevados indicando maior morbidade psicológica. Os participantes foram classificados como possíveis casos de TMC, utilizando o ponto de corte $4 / 5$, conforme validação para a amostra do SPAH 15.

Informações sobre características individuais dos participantes incluíram: sexo (feminino ou masculino); faixa etária $(<40,40-64,65-69,70-74$, 75 ou mais); estado civil (nunca casou, casado/ vive junto, viúvo, divorciado/separado); escolaridade (nenhuma, mínima, 1o Grau completo, 2o Grau completo, Superior completo/Pós-graduação); e ocupação (se tem ou não tem).

Informações sobre características dos domicílios incluíram: tipo de domicílio (próprio quitado, próprio em aquisição, alugado, cedido, invadido); número de pessoas e de crianças; número de cômodos; renda familiar em reais (0-720, 721-1.440, 1.441-2.160, 2.161 ou mais); número de bens materiais (TV, freezer, telefone e carro); e presença de saneamento básico. A variável aglomeração foi gerada pela razão entre número de moradores e número de cômodos da residência $(\leq 0,5,>0,5 \mathrm{e} \leq 1,>1)$.

Setores censitários, conforme definidos pelo Instituto Brasileiro de Geografia e Estatística (IBGE) 16, foram utilizados como indicadores de área de residência.

\section{Análise estatística}

As análises foram realizadas com o software Stata SE 10.0 (Stata Corp., College Station, Estados Unidos). Na etapa bivariada da análise, foram examinadas as associações entre TMC e as variáveis de exposição individuais e de características dos domicílios, por meio de testes de qui-quadrado de Pearson ou teste de qui-quadrado para tendência linear, quando a variável de exposição tinha mais de dois níveis ordenados. Foram estimados odds ratios (OR) com respectivos intervalos de $95 \%$ de confiança (IC95\%).
Na etapa multivariada, foi realizada uma análise multinível, técnica estatística apropriada para investigar, no mesmo modelo, associações entre exposições no nível do indivíduo e em níveis hierarquicamente superiores, que caracterizam o contexto onde vive o indivíduo, e o desfecho de interesse 11,17. Indivíduos pertencentes a um mesmo grupo tendem a ser mais parecidos entre si em relação às exposições e aos desfechos relacionados à saúde do que indivíduos de grupos diferentes. No presente estudo, a estrutura hierárquica foi definida da seguinte forma: indivíduos (nível um); domicílios (nível dois); e setores censitários (nível três). Os valores de $\mathrm{p}$ foram baseados no teste de verossimilhança. Nossa estratégia de análise consistiu em primeiro gerar um modelo de componentes de variância (modelo nulo) para identificarmos a possível contribuição dos setores censitários (nível 3) e dos domicílios (nível 2). O próximo passo foi gerar um modelo (modelo 1) incluindo todas as variáveis relacionadas aos indivíduos. Por fim, foi gerado um novo modelo (modelo 2) acrescentando-se ao modelo 1 as variáveis do nível dos domicílios. Os modelos multinível foram construídos usando o comando GLLAMM do Stata 18.

\section{Resultados}

As características sociodemográficas e dos domicílios dos participantes estão apresentadas nas Tabelas 1 e 2. A média de idade foi de 61 anos, $64 \%$ dos participantes eram mulheres, $74 \%$ tinham escolaridade mínima ou nenhuma escolaridade, $56,4 \%$ eram casados, e 63,9\% não trabalhavam. Em relação aos domicílios, $70 \%$ dos participantes possuíam residência quitada ou em aquisição, a taxa de aglomeração foi maior que 0,5 para $77 \%$ dos participantes, e, para $32 \%$, havia, pelo menos, uma criança no domicílio, 37,8\% dos participantes moravam em domicílios que tinham 4 ou mais itens, $85 \%$ dos participantes viviam em domicílios com banheiro, e 24,5\% viviam em domicílios com renda de, no máximo, $\mathrm{R} \$ 720,00$.

A prevalência de TMC foi de 43,1\% (IC95\%: 41,1-45,1). Em relação às características individuais, TMC associou-se a sexo, faixa etária, escolaridade e ocupação (Tabela 1). Mulheres e indivíduos na faixa etária de 40 a 64 anos tiveram maior chance de apresentar TMC. Quanto maior a escolaridade dos participantes, menor a prevalência de TMC. Participantes que tinham ocupação apresentaram menor prevalência de TMC do que os que não trabalhavam. O estado civil não se mostrou associado com a prevalência de TMC.

Em relação às características dos domicílios, nível de aglomeração, presença de crianças no 
Prevalência de transtornos mentais comuns (TMC) de acordo com características sociodemográficas, odds ratios (OR), intervalos de 95\% de confiança (IC95\%) e valores de p. São Paulo, Brasil, 2003-2005 (N = 2.366).

\begin{tabular}{|c|c|c|c|c|c|c|c|}
\hline \multirow[t]{2}{*}{ Características } & \multicolumn{2}{|c|}{ Total } & \multicolumn{2}{|c|}{ TMC } & \multirow[t]{2}{*}{ OR } & \multirow[t]{2}{*}{ IC95\% } & \multirow[t]{2}{*}{ Valor de $p$} \\
\hline & $\mathrm{n}$ & $\%$ & $n$ & $\%$ & & & \\
\hline \multicolumn{8}{|l|}{ Sexo } \\
\hline Masculino & 851 & 36,0 & 226 & 26,6 & 1,00 & - & \\
\hline Feminino & 1.515 & 64,0 & 794 & 52,4 & 3,05 & $2,52-3,68$ & $<0,001$ \\
\hline \multicolumn{8}{|l|}{ Faixa etária (anos) } \\
\hline$<40$ & 314 & 13,3 & 146 & 46,5 & 1,00 & - & \\
\hline $40-64$ & 625 & 26,4 & 344 & 55,0 & 1,41 & $1,07-1,85$ & \\
\hline $65-69$ & 648 & 27,4 & 217 & 33,5 & 0,58 & $0,44-0,76$ & \\
\hline $70-74$ & 375 & 15,8 & 137 & 36,5 & 0,66 & $0,49-0,90$ & \\
\hline 75 ou mais & 404 & 17,1 & 176 & 43,6 & 0,89 & $0,66-1,20$ & $<0,001$ \\
\hline \multicolumn{8}{|l|}{ Escolaridade } \\
\hline Nenhuma & 590 & 24,9 & 283 & 48,0 & 1,00 & - & \\
\hline Mínima & 1.161 & 49,1 & 506 & 43,6 & 0,84 & $0,70-1,02$ & \\
\hline 1ㅇ Grau completo & 254 & 10,7 & 107 & 42,1 & 0,79 & $0,59-1,06$ & \\
\hline 2o Grau completo & 290 & 12,3 & 110 & 37,9 & 0,66 & $0,50-0,88$ & \\
\hline Superior completo/Pós-graduação & 71 & 3,0 & 14 & 19,7 & 0,27 & $0,14-0,49$ & $<0,001$ \\
\hline \multicolumn{8}{|l|}{ Estado civil } \\
\hline Nunca casou & 355 & 15,0 & 160 & 45,1 & 1,00 & - & \\
\hline Casado/Vive junto & 1.336 & 56,4 & 557 & 41,7 & 0,87 & $0,69-1,10$ & \\
\hline Viúvo & 406 & 17,2 & 173 & 42,6 & 0,90 & $0,68-1,21$ & \\
\hline Divorciado/Separado & 269 & 11,4 & 130 & 48,3 & 1,14 & $0,83-1,57$ & 0,20 \\
\hline \multicolumn{8}{|l|}{ Ocupação } \\
\hline Não & 1.511 & 63,9 & 707 & 46,8 & 1,00 & - & \\
\hline Sim & 855 & 36,1 & 313 & 36,6 & 0,66 & $0,55-0,78$ & $<0,001$ \\
\hline
\end{tabular}

domicílio, quantidade de bens materiais, saneamento básico e renda familiar mostraram-se associados à prevalência de TMC. Quanto maior a taxa de aglomeração da residência, maior a proporção de pessoas que apresentavam TMC. A prevalência de TMC foi maior em domicílios com crianças. Quanto menor o número de itens no domicílio, maior a prevalência de TMC. Presença de saneamento básico e maior renda familiar associaram-se negativamente à prevalência de TMC.

Na análise multinível, o modelo nulo apresentou pequenas proporções da variância nos níveis dos setores e dos domicílios, com valores de 0,034 e 0,465, respectivamente (Tabela 3). No modelo 1, observamos um aumento da variância no nível do domicílio para 0,862 , após ajuste para características individuais. $\mathrm{O}$ modelo 2 , que incluiu variáveis indicadoras de características dos domicílios, apresentou variância de 0,011 para o nível 3 (setores) e 0,803 para o nível 2 (domicílio), uma diferença pouco expressiva em relação ao modelo 1. Faixa de renda familiar e aglomeração mantiveram-se independentemente associadas à prevalência de TMC (Tabela 4), a primeira de forma negativa e a segunda de forma positiva.

\section{Discussão}

A presente análise visou estudar a associação de características individuais, do domicílio e área de residência com a prevalência de TMC, em uma amostra de base populacional de áreas socioeconomicamente desfavorecidas do Município de São Paulo. A população estudada foi predominantemente idosa, feminina, sem escolaridade e sem ocupação. No nível individual, ser mulher, ter entre 40 e 64 anos, ter nenhuma ou escolaridade mínima e não ter ocupação mostraram-se independentemente associados à prevalência de TMC. No nível do domicílio, renda familiar e nível de aglomeração também se mostraram associados à prevalência de TMC, independentemente das características individuais. Não observamos associação entre área de residência, re- 
Tabela 2

Prevalência de transtornos mentais comuns (TMC) de acordo com características do domicílio, odds ratios (OR), intervalos de 95\% de confiança (IC95\%) e valores de p. São Paulo, Brasil, 2003-2005 (N = 2.366).

\begin{tabular}{|c|c|c|c|c|c|c|c|}
\hline \multirow[t]{2}{*}{ Características } & \multicolumn{2}{|c|}{ Total } & \multicolumn{2}{|c|}{ TMC } & \multirow[t]{2}{*}{ OR } & \multirow[t]{2}{*}{ IC95\% } & \multirow[t]{2}{*}{ Valor de $p$} \\
\hline & $\mathrm{n}$ & $\%$ & $\mathrm{n}$ & $\%$ & & & \\
\hline \multicolumn{8}{|l|}{ Tipo de domicílio } \\
\hline Próprio (quitado) & 1.297 & 54,8 & 523 & 40,3 & 1,00 & - & \\
\hline Próprio (em aquisição) & 357 & 15,1 & 162 & 45,4 & 1,23 & $0,97-1,56$ & \\
\hline Alugado & 124 & 5,2 & 57 & 46,0 & 1,26 & $0,87-1,82$ & \\
\hline Cedido & 157 & 6,7 & 75 & 47,8 & 1,35 & $0,97-1,89$ & \\
\hline Invadido & 431 & 18,2 & 203 & 47,1 & 1,32 & $1,06-1,64$ & 0,051 \\
\hline \multicolumn{8}{|l|}{ Aglomeração } \\
\hline$\leq 0,5$ & 545 & 23,1 & 196 & 36,0 & 1,00 & - & \\
\hline$>0,5$ e $\leq 1,0$ & 1.179 & 49,8 & 501 & 42,5 & 1,32 & $1,07-1,62$ & \\
\hline$>1,0$ & 642 & 27,1 & 323 & 50,3 & 1,80 & $1,42-2,28$ & $<0,001$ \\
\hline \multicolumn{8}{|l|}{ Mora com crianças? } \\
\hline Não & 1.611 & 68,1 & 659 & 40,9 & 1,00 & - & \\
\hline Sim & 755 & 31,9 & 361 & 47,8 & 1,32 & $1,11-1,58$ & 0,002 \\
\hline \multicolumn{8}{|l|}{ Bens materiais (itens) } \\
\hline Até 2 & 364 & 15,4 & 182 & 50,0 & 1,00 & - & \\
\hline 3 & 1.107 & 46,8 & 502 & 45,4 & 0,83 & $0,65-1,05$ & \\
\hline 4 ou mais & 895 & 37,8 & 336 & 37,5 & 0,60 & $0,47-0,77$ & $<0,001$ \\
\hline \multicolumn{8}{|l|}{ Saneamento básico } \\
\hline Sem banheiro & 354 & 15,0 & 175 & 49,4 & 1,00 & - & \\
\hline Com banheiro & 2.012 & 85,0 & 845 & 42,0 & 0,74 & $0,59-0,93$ & 0,009 \\
\hline \multicolumn{8}{|l|}{ Renda familiar (R\$) * } \\
\hline $0-720$ & 577 & 24,4 & 282 & 48,9 & 1,00 & - & \\
\hline $721-1.440$ & 841 & 35,6 & 389 & 46,3 & 0,90 & $0,73-1,11$ & \\
\hline $1.441-2.160$ & 426 & 18,0 & 183 & 43,0 & 0,79 & $0,61-1,01$ & \\
\hline 2.161 ou mais & 512 & 21,6 & 162 & 31,6 & 0,48 & $0,38-0,62$ & $<0,001$ \\
\hline
\end{tabular}

* Dez valores faltantes.

Tabela 3

Estimativas dos efeitos aleatórios dos modelos de regressão logística multinível. São Paulo, Brasil, 2003-2005.

\begin{tabular}{cccc}
\hline & $\begin{array}{c}\text { Modelo nulo } \\
\text { Variância (EP) }\end{array}$ & $\begin{array}{c}\text { Modelo 1* } \\
\text { Variância (EP) }\end{array}$ & $\begin{array}{c}\text { Modelo 2 ** } \\
\text { Variância (EP) }\end{array}$ \\
\hline Nível 3 (setor) & $0,034(0,041)$ & $0,008(0,037)$ & $0,011(0,037)$ \\
Nível 2 (domicílio) & $0,465(0,158)$ & $0,862(0,219)$ & $0,803(0,223)$ \\
Valor de p & & $<0,001$ & $<0,001$ \\
\hline
\end{tabular}

EP: erro-padrão.

* Modelo com variáveis do nível individual;

** Modelo com variáveis dos níveis individual e de domicílio.

presentada pelos setores censitários, e prevalência de TMC. Uma parte da variância foi explicada pelo nível do domicílio, mesmo após ajuste para características individuais.
No presente estudo, encontramos associações de características individuais e prevalência de TMC consistentes com a literatura internacional: ser do sexo feminino; ser mais jovem; ter 
Estimativas dos efeitos fixos do modelo de regressão logística multinível. São Paulo, Brasil, 2003-2005.

\begin{tabular}{|c|c|c|c|c|c|c|}
\hline \multirow[t]{2}{*}{ Características } & \multicolumn{3}{|c|}{ Modelo 1 * } & \multicolumn{3}{|c|}{ Modelo 2 ** } \\
\hline & OR & IC95\% & Valor de $p$ & OR & IC95\% & Valor de $p$ \\
\hline \multicolumn{7}{|l|}{ Sexo } \\
\hline Masculino & 1,00 & - & & 1,00 & - & \\
\hline Feminino & 2,97 & $2,45-3,60$ & $<0,001$ & 3,00 & $2,48-3,64$ & $<0,001$ \\
\hline \multicolumn{7}{|l|}{ Faixa etária (anos) } \\
\hline$<40$ & 1,00 & - & & 1,00 & - & \\
\hline $40-64$ & 0,98 & $0,66-1,46$ & & 1,15 & $0,77-1,74$ & \\
\hline $65-69$ & 0,35 & $0,23-0,54$ & & 0,43 & $0,27-0,66$ & \\
\hline $70-74$ & 0,36 & $0,22-0,59$ & & 0,45 & $0,28-0,73$ & \\
\hline 75 ou mais & 0,47 & $0,30-0,74$ & 0,001 & 0,59 & $0,38-0,92$ & $<0,001$ \\
\hline \multicolumn{7}{|l|}{ Escolaridade } \\
\hline Nenhuma & 1,00 & - & & 1,00 & - & \\
\hline Mínima & 0,83 & $0,67-1,03$ & & 0,94 & $0,76-1,17$ & \\
\hline 1ㅇ Grau completo & 0,54 & $0,36-0,82$ & & 0,67 & $0,44-1,00$ & \\
\hline 2o Grau completo & 0,41 & $0,26-0,66$ & & 0,54 & $0,34-0,86$ & \\
\hline Superior completo/Pós-graduação & 0,16 & $0,07-0,35$ & $<0,001$ & 0,28 & $0,13-0,63$ & 0,001 \\
\hline \multicolumn{7}{|l|}{ Ocupação } \\
\hline Não & 1,00 & - & & 1,00 & - & \\
\hline Sim & 0,62 & $0,49-0,78$ & $<0,001$ & 0,63 & $0,50-0,79$ & 0,001 \\
\hline \multicolumn{7}{|l|}{ Aglomeração } \\
\hline$\leq 0,5$ & & & & 1,00 & - & \\
\hline$>0,5$ e $\leq 1,0$ & & & & 1,19 & $0,89-1,60$ & \\
\hline$>1,0$ & & & & 1,52 & $1,03-2,24$ & 0,014 \\
\hline \multicolumn{7}{|l|}{ Mora com crianças? } \\
\hline Não & & & & 1,00 & - & \\
\hline Sim & & & & 1,05 & $0,80-1,39$ & 0,787 \\
\hline \multicolumn{7}{|l|}{ Bens materiais (itens) } \\
\hline Até 2 & & & & 1,00 & - & \\
\hline 3 & & & & 0,96 & $0,68-1,36$ & \\
\hline 4 ou mais & & & & 0,82 & $0,56-1,21$ & 0,292 \\
\hline \multicolumn{7}{|l|}{ Saneamento básico } \\
\hline Sem banheiro & & & & 1,00 & - & \\
\hline Com banheiro & & & & 0,85 & $0,58-1,25$ & 0,679 \\
\hline \multicolumn{7}{|l|}{ Renda familiar (R\$) } \\
\hline $0-720$ & & & & 1,00 & - & \\
\hline $721-1.440$ & & & & 1,05 & $0,79-1,38$ & \\
\hline $1.441-2.160$ & & & & 0,99 & $0,70-1,42$ & \\
\hline 2.161 ou mais & & & & 0,61 & $0,43-0,86$ & 0,004 \\
\hline
\end{tabular}

* Modelo com variáveis do nível individual;

** Modelo com variáveis dos níveis individual e de domicílio.

pouca escolaridade; não ter ocupação ${ }^{8,19}$. Além disso, algumas características dos domicílios (aglomeração e renda familiar) mostraram-se independentemente associadas à prevalência de TMC. Em análise de amostra representativa da população adulta do Reino Unido, Weich et al. 11 também observaram associação entre renda do domicílio e prevalência de TMC, independentemente de características individuais, porém não encontraram associação entre aglomeração e prevalência de TMC. Esses resultados indicam que, além de condições socioeconômicas desfavoráveis individuais aumentarem o risco de TMC, há uma contribuição extra para esse 
risco em função das condições socioeconômicas do microambiente; nesse caso, representado pelo nível de domicílio no qual o indivíduo está inserido.

Outro resultado significativo que encontramos foi mostrar que parte da variância relacionada à prevalência de TMC foi associada ao nível dos domicílios, mesmo após o controle para características individuais e dos domicílios, resultado similar aos de investigações prévias 10,11. Isso sugere que possivelmente há outros elementos relacionados ao microambiente onde os indivíduos estão inseridos, não mensurados pelas variáveis incluídas nesta análise (aglomeração e renda familiar), que influenciam a probabilidade desses indivíduos apresentarem TMC.

O presente estudo apresenta limitações. Os setores censitários do IBGE, que têm como finalidade organizar a coleta de dados dos censos populacionais, não são a melhor forma de agrupar pessoas de acordo com seu contexto social 16. De fato, a análise multinível não evidenciou variância significativa no nível dos setores censitários, o que talvez se deva à pouca variabilidade entre eles, sugerindo a necessidade de utilizar agrupamentos populacionais com maior heterogeneidade entre eles para investigar a contribuição de área de residência na saúde mental. Além disso, estudos que utilizaram área de residência como indicador do contexto social, como as unidades eleitorais do Reino Unido 11,20,21, os setores censi- tários de áreas urbanas do Canadá 22 e os distritos de Amsterdã (Holanda) 23, mostraram que esse nível contribuía com uma pequena proporção da variância total relacionada à prevalência de TMC, entre 1 e 3\% do total da variância. A amostra examinada no presente estudo possuía poucas pessoas por domicílio, em geral duas, o que pode ter limitado o poder estatístico das análises. O questionário SRQ-20 não é um instrumento diagnóstico, sendo originalmente construído para ser utilizado como instrumento de rastreamento para TMC. Entretanto, de acordo com a validação na amostra investigada, o ponto de corte 4/5 apresentou boa especificidade e sensibilidade em relação a outras validações desse instrumento em populações adultas 15 .

A presente investigação evidenciou uma contribuição do microambiente domiciliar na prevalência de TMC, em parte relacionada às condições socioeconômicas desse microambiente. Políticas públicas voltadas à melhoria das condições de vida das famílias, por meio de programas de renda mínima ou políticas de inclusão social, poderão ter um impacto positivo na redução do sofrimento psíquico nas populações. Pesquisas futuras também devem se concentrar na investigação do possível papel do contexto social, como vizinhança, coesão social e infraestrutura, utilizando divisões geográficas adequadas para contribuir na redução das iniquidades em saúde mental.

\section{Resumen}

El contexto social puede tener un importante papel en la etiología de los trastornos mentales y en su prevalencia. El objetivo del presente estudio fue investigar factores de riesgo que contribuyen a la prevalencia de trastornos mentales comunes (TMC), considerando distintos niveles contextuales: individuo, domicilio y área de censo. Para ello se utilizó una muestra de base poblacional con 2.366 individuos, participantes del São Paulo Ageing \& Health Study. La presencia de TMC fue identificada por el instrumento SRQ-20. Sexo, edad, escolaridad y ocupación fueron las características individuales asociadas a la prevalencia de TMC.
Los modelos de regresión logística multinivel mostraron que una parte de la variancia en la prevalencia de TMC se asoció al nivel de domicilio, con asociaciones entre aglomeración, renta de la familia y prevalencia de TMC, incluso después del control para características individuales. Estos resultados sugieren que las características del ambiente donde viven las personas contribuyen a su salud mental.

Trastornos Mentales; Salud Mental; Análisis Multinivel 


\section{Colaboradores}

L. M. S. Coutinho contribuiu com a concepção e projeto, análise e interpretação dos dados, redação do artigo e aprovação final da versão a ser publicada. A. Matijasevich contribuiu com a análise e interpretação dos dados, redação do artigo e aprovação final da versão a ser publicada. M. Scazufca contribuiu com a revisão crítica relevante do conteúdo intelectual e aprovação final da versão a ser publicada. P. R. Menezes contribuiu com a concepção e projeto, análise e interpretação dos dados, revisão crítica relevante do conteúdo intelectual e aprovação final da versão a ser publicada.

\section{Agradecimentos}

Os autores agradecem à Renata Nunes dos Santos pela elaboração dos bancos de dados do SPAH. Ao Welcome Trust, do Reino Unido (GR066133MA), e CNPq.

\section{Referências}

1. Prince M, Patel V, Saxena S, Maj M, Maselko J, Phillips MR, et al. No health without mental health. Lancet 2007; 370:859-77.

2. World Health Organization. Towards a common language for functioning disability and health ICF. Geneva: World Health Organization; 2002.

3. Associação Psiquiátrica Americana. Manual diagnóstico e estatístico de transtornos mentais (DSMIV), 4a Ed. Porto Alegre: Editora Artmed; 1994.

4. Organização Mundial da Saúde. Classificação estatística internacional de doenças e problemas relacionados à saúde (CID-10), 10ạ revisão. 8ạ Ed. São Paulo: Edusp; 2000.

5. Goldberg D, Huxley P. Common mental disorders: a bio-social model. London: Routledge; 1992.

6. Weich S, Lewis G, Jenkins SP. Income inequality and the prevalence of common mental disorders in Britain. Br J Psychiatry 2001; 178:222-7.
7. Skapinakis P, Lewis G, Araya R, Jones K, Williams G. Mental health inequalities in Wales, UK: multilevel investigation of the effect of area deprivation. Br J Psychiatry 2005; 186:417-22.

8. Araya R, Montgomery A, Rojas G, Fritsch R, Solis J, Signorelli A, et al. Common mental disorders and the built environment in Santiago, Chile. Br J Psychiatry 2007; 190:394-401.

9. Araya R, Dunstan F, Playle R, Thomas H, Palmer S, Lewis G. Perceptions of social capital and the built environment and mental health. Soc Sci Med 2006; 62:3072-83.

10. Thomas H, Weaver N, Patterson J, Jones P, Bell T, Playle R, et al. Mental health and quality of residential environment. Br J Psychiatry 2007; 191:500-5.

11. Weich S, Holt G, Twigg L, Lewis G. Geographic variation in the prevalence of common mental disorders in Britain: a multilevel investigation. Am J Epidemiol 2003; 157:730-7. 
12. Taschner SP, Bógus LM. São Paulo: o caleidoscópio urbano. São Paulo Perspec 2001; 15:31-44.

13. Scazufca M, Menezes PR, Vallada HP, Crepaldi AL, Pastor-Valero M, Coutinho LM, et al. High prevalence of dementia among older adults from poor socioeconomic backgrounds in Sao Paulo, Brazil. Int Psychogeriatr 2008; 20:394-405.

14. Harding TW, Arango MV, Baltazar J, Climent CE, Ibrahim HH, Ladrido-Ignacio L, et al. Mental disorders in primary health care: a study of their frequency and diagnosis in four developing countries. Psychol Med 1980; 10:231-41.

15. Scazufca M, Menezes PR, Vallada H, Araya R. Validity of the self reporting questionnaire-20 in epidemiological studies with older adults. Soc Psychiatry Psychiatr Epidemiol 2009; 44:247-54.

16. Instituto Brasileiro de Geografia e Estatística. Um panorama da saúde no Brasil: acesso e utilização dos serviços, condições e fatores de risco e proteção à saúde - 2008. Rio de Janeiro: Instituto Brasileiro de Geografia e Estatística; 2010.

17. O'Campo P. Invited commentary: advancing theory and methods for multilevel models of residential neighborhoods and health. Am J Epidemiol 2003; 157:9-13.
18. Habe-Hesketh S, Skrondal A, Pickles A. GLLAMM manual. Berkeley; University of California; 2004. (U.C. Berkeley Division of Biostatistics Working Paper Series. Working Paper, 160.)

19. Weich S, Lewis G. Poverty, unemployment, and common mental disorders: population based cohort study. BMJ 1998; 317:115-9.

20. Wainwright NW, Surtees PG. Area and individual circumstances and mood disorder prevalence. $\mathrm{Br} \mathrm{J}$ Psychiatry 2004; 185:227-32.

21. Fone DL, Lloyd K, Dunstan FD. Measuring the neighbourhood using UK benefits data: a multilevel analysis of mental health status. BMC Public Health 2007; 7:69.

22. Matheson FI, Moineddin R, Dunn JR, Creatore MI, Gozdyra P, Glazier RH. Urban neighborhoods, chronic stress, gender and depression. Soc Sci Med 2006; 63:2604-16.

23. Reijneveld SA, Schene AH. Higher prevalence of mental disorders in socioeconomically deprived urban areas in The Netherlands: community or personal disadvantage? J Epidemiol Community Health 1998; 52:2-7.

Recebido em 09/Out/2013

Versão final reapresentada em 16/Jan/2014

Aprovado em 17/Fev/2014 